\title{
Day-ahead forecasting of grid carbon intensity in support of HVAC plant demand response decision-making to reduce carbon emissions
}

\author{
Gordon Lowry
}

School of the Built Environment and Architecture,

London South Bank University,

London SE1 0AA, UK

Email: gordon.lowry@1sbu.ac.uk

\begin{abstract}
Electrical HVAC loads in buildings are suitable candidates for use in demand response activity. This paper demonstrates a method to support planned demand response actions intended explicitly to reduce carbon emissions. Demand response is conventionally adopted to aid the operation of electricity grids and can lead to greater efficiency; here it is planned to target times of day when electricity is generated with high carbon intensity. Operators of HVAC plant and occupants of conditioned spaces can plan when to arrange shutdown of plant once they can foresee the opportune time of day for carbon saving. It is shown that the carbon intensity of the mainland UK electricity grid varies markedly throughout the day, but that this tends to follow daily and weekly seasonal patterns. To enable planning of demand response, 24-hour ahead forecast models of grid carbon intensity are developed that are not dependent on collecting multiple exogenous data sets. In forecasting half-hour periods of high carbon intensity either linear autoregressive or non-linear ANN models can be used, but a daily seasonal autoregressive model is shown to provide a $20 \%$ improvement in carbon reduction.
\end{abstract}

\section{Practical application}

The forecast method demonstrated in the paper would enable building operators to plan demand response activity to target times of high carbon intensity on the UK electricity grid. The method would be easy to implement as the only data required is publicly available.

\section{Keywords:}

demand response, carbon intensity, autoregressive model, artificial neural network 


\section{Introduction}

This paper sets out to demonstrate how, by developing a day-ahead forecast of the timevarying carbon intensity of grid electricity, carbon saving from demand response can be increased. The proposal is to help plan for electrical load reduction to coincide with high carbon intensity. Electricity generation in the UK has been highly dependent on coal and oil for its main source of fuel, but these power plants are progressively being replaced by less carbon intensive fuels namely gas, nuclear and renewable energy sources like solar, hydro, wind and biomass. ${ }^{1}$ Such efforts may achieve the target of $80 \%$ reduction in $\mathrm{CO}_{2}$ emission by $2050,{ }^{2}$ but there are more immediate actions needed. In situations where carbon emissions reductions are to be achieved from reducing electricity use, the relevant carbon intensity of the electricity is significant. Ordinarily, total, national demand for electricity varies considerably diurnally and seasonally. Typically, UK demand rises at the start of the working day reaching a plateau between 9:00 and 16:00, and rising again between 16:00 and 17:30 owing to lighting loads and increased domestic demand. ${ }^{3}$ Seasonal variation is often weather dependent and UK demand tends to increase in the winter.

Electricity is generated from a mixture of fuels. For each fuel used in the UK there are published emissions factors quantifying the equivalent carbon dioxide emissions per unit of electricity $(\mathrm{kg} / \mathrm{kWh})$. Individual generators with the same fuel may have different efficiencies so the factors are averaged for each fuel. Over a long timescale in which generators are re-engineered or replaced these factors will slowly change but they change little over the time scales of interest in this paper. The system-average carbon intensity can be calculated from a weighted sum of the emission factors for fuels according to their contribution to the mix. As a result of variation in demand, the mixture of fuels used in generation continually varies, and so does the electricity carbon intensity.

There has been some debate about how best to evaluate the relevant carbon intensity for particular forms of electricity demand reduction. ${ }^{4-6}$ In particular, noting that a reduction in energy use might change the proportion of fuels used since some plant will respond more readily to load change than others. Thus, marginal factors can be estimated that attribute emissions reductions to particular demand reductions by predicting how this change would affect different generators. How this should be done would depend on the timescales involved in the demand reduction.

To quantify emissions reductions from policy developments effecting overall long-term change, one needs to consider how the mix of generators could be permanently altered, and relevant carbon intensity would be that of new generation plant not built or old plant decommissioned. ${ }^{7,8}$ Particular technological innovations might tend to concentrate demand reduction at certain times of day, and the intensity for implementing these may be calculated from an average for that time of day. ${ }^{5,6,9}$ For short-term measures, such as daily demand response actions, it has been suggested that the marginal intensity might be inferred from a knowledge of the merit order in which generators are dispatched. Then, the last generator to be dispatched is assumed to be the one that reduces output as a result of the marginal demand reduction and, thus, its emission factor 
is taken to be the marginal grid carbon intensity. Ascertaining the merit order is not straightforward, however, and must be estimated or inferred from historical data. ${ }^{4,9-11}$ Even if the merit order was known, this is not a realistic approach for larger systems where more than one type of generator might be dispatched at a time, and dispatch can be determined by other factors apart from merit order. ${ }^{12}$ Alternatively, instantaneous marginal values might be calculated from current grid activity. The ratio of simultaneous changes in carbon emissions and changes in demand represent a marginal value, and have been used for a simple small system in Singapore. ${ }^{13}$ Unfortunately this would be misleading if the change in load distribution across generators changes for reasons other than demand. This is likely in a larger system working with various operational constraints, ${ }^{14}$ and it could not then be known how to attribute a marginal change in demand to any particular generator.

In this paper the concern is for immediate-term changes in load in single buildings, which are much smaller than the regular fluctuation in demand on generators, and thus making no discernible difference to the generation fuel mix. ${ }^{5}$ As calculating an appropriate marginal effect is not possible, the overall grid carbon intensity will be used rather than an instantaneous marginal value. Of more importance here is that the precise time of the demand reduction is to be determined, at the risk of underestimating the emissions reductions. ${ }^{4}$

Demand response (DR) is usually advocated for electricity grid management and entails electrical loads being disconnected at times determined by the grid operator or through pricing signals, ${ }^{15}$ and this may reduce and smooth demand. ${ }^{16}$ Large loads are required to satisfy DR contracts, so the processes are employed by large consumers, or aggregators that can ensure many smaller consumers act in concert. ${ }^{17}$ This also helps in managing voltage profiles on the network. ${ }^{18}$ Consumers who have agreed to support the network by reducing their electricity demand by a predefined amount, either switch off low priority loads or use embedded generation plant. ${ }^{19}$ Modifying the consumption pattern of electricity consumers could lead to emissions reductions if load is rescheduled to times to avoid the need to use generator plant with high emission factor. ${ }^{20}$ Suitable candidate loads in buildings for use in DR must be able to cease for limited periods without adversely affecting occupants or the activity in the building. Electrical heating, ventilation and air-conditioning (HVAC) plant commonly installed in commercial buildings can exploit the thermal storage inherent in the building air and fabric, and permit suspended operation for short periods. ${ }^{21}$ In order to maintain acceptable internal conditions, the plant would have to make up the heating or cooling at other times with lower carbon intensity. The net reduction in emissions would depend on the particular characteristics of the plant and building, as would the acceptable duration of any cessation in plant operation. The particular response of a system identified for DR would need to be trialed on-site.

Outside of formal contractual arrangements for DR, any organisation might elect to reduce load locally. This might be implemented as part of a number of measures to reduce carbon emissions, but DR would provide an immediate effect. To reduce emissions this should be done at a time when the grid electricity is generated from the most carbon-intensive fuels. Services are currently offered that track the instantaneous 
carbon intensity for making immediate power use decisions. ${ }^{22,23}$ However, for most business users there would be a need to plan ahead for the DR event each day, and the intention of this paper is to demonstrate a method to support the decision making 24 hours ahead. Values of average carbon intensity for the UK grid are published for carbon auditing purposes ${ }^{24}$ but these data do not consider the daily variation resulting from the continual change in fuel mix used for electricity generation. Instead, a predictive model is proposed to forecast peaks in the carbon intensity a day ahead.

\section{Forecasting Methods}

The usual objective for modelling grid activity is to forecast demand, and various methods have been demonstrated that could be candidates for forecasting the corresponding carbon intensity time series. There are numerous load forecasting techniques recommended ${ }^{25-28}$ that are based on different requirements, and complexities, but many require input data not readily available.

Grey models proposed by Deng ${ }^{29}$ have been used for very short-term demand forecasts $^{30}$ and longer term forecasting of carbon emissions. ${ }^{31}$ Grey modelling allows for a small number of steps ahead but uses little historical data. Grey theory has been widely used in forecasting studies because of its higher forecasting accuracy when compared with other forecasting techniques ${ }^{32-34}$ but it is most effective with monotonically varying time series.

Autoregressive integrated moving average (ARIMA) models have been extensively used in forecasting because few assumptions need to be made. ${ }^{35}$ Non-linear autoregressive models with exogenous input (NARX) have been shown to perform well, at least with one-step ahead forecasts. ${ }^{36}$ Artificial neural networks (ANN) have been extensively studied and used in time-series forecasting. The major advantage of neural networks is their flexible non-linear modelling capability. Artificial neural networks (ANN) have been used with hourly ${ }^{37}$ and monthly ${ }^{38.39}$ data. They have also been used to examine the energy use in buildings. ${ }^{40}$ Scenarios of future carbon emissions per unit GDP have been explored using ANN. ${ }^{41}$ However, any superiority of ANN-based methods over linear methods are usually demonstrated in very short-term forecasting, e.g. one-step ahead. The challenge in this paper is to achieve 24-hour ahead forecasting on half-hourly data, thus 48-step ahead.

Grid demand forecasting will typically rely on the availability of exogenous inputs, such as weather forecasts. For individual buildings, temperature data have been used to aid dispatch decision-making for the use of combined heat and power plant. ${ }^{42}$ There may be a number of time-varying drivers that influence the grid carbon intensity, and whose inclusion should improve the predictive power of any model. Critically, the building operator is unlikely to have access to current data for these, and the proposal is to ascertain the extent to which sufficient useful information might be extracted from the carbon intensity time series alone. There is freely available several years' worth of data on the demand and fuel mix used for the mainland UK grid, e.g. Gridwatch, ${ }^{43}$ and this will be modelled using the ANN and autoregressive approaches that have worked well in demand forecasting. 


\section{Sources of grid carbon intensity data}

Data for the mainland UK grid carbon intensity can be calculated from the data published by Gridwatch ${ }^{43}$ derived from the UK Balancing Mechanism Reporting Service. These data include the five-minute average for electricity demand in GW, and how this is distributed across the major fuels used in the UK. For individual fuels, their carbon emission factors are available ${ }^{44}$ as shown in Table 1 , and can be used to ascertain the overall carbon intensity for each time interval. For this study, the large number of small contributions from transnational interconnectors and unmetered renewable sources have not been allowed for. Currently these represent only a small proportion of the total and determining emission factors for these would be unreliable and therefore carbon intensity is calculated without these.

\begin{tabular}{|lc|}
\hline Fuel & $\begin{array}{c}\text { carbon emission factors } \\
\left(\mathrm{kgCO}_{2} / \mathrm{kWh}\right)\end{array}$ \\
\hline COAL & 0.870 \\
\hline NUCLEAR & 0.016 \\
\hline WIND & 0.011 \\
\hline GAS (combined cycle) & 0.487 \\
\hline GAS (open cycle) & 0.651 \\
\hline HYDRO & 0.020 \\
\hline OIL & 0.650 \\
\hline
\end{tabular}

\section{Table 1. Carbon emission factors for electricity generated from specific fuels ${ }^{44}$}

For this study, data were downloaded for the period 29 May 2011 to 22 January 2018. These data are at 5-minute intervals and comprise almost 700000 time steps. In order to reduce the data processing time these were aggregated into half-hourly data. The half-hourly carbon intensity was calculated using the weighted sum of carbon emission factors from Table 1 and this time series is shown in Fig. 1. Half-hourly data is the time resolution commonly used for electricity data, and represents a sensible delay before restarting HVAC plant that has been shut down as a demand response. Missing data values were estimated using linear interpolation. This left a time series of more than 110000 values, sufficient for the training of an artificial neural network. This data sequence was then partitioned to allow the data from 1 July 2017 to the end to be held back for model validation. This captured a half-year incorporating summer and winter grid demand variation. 


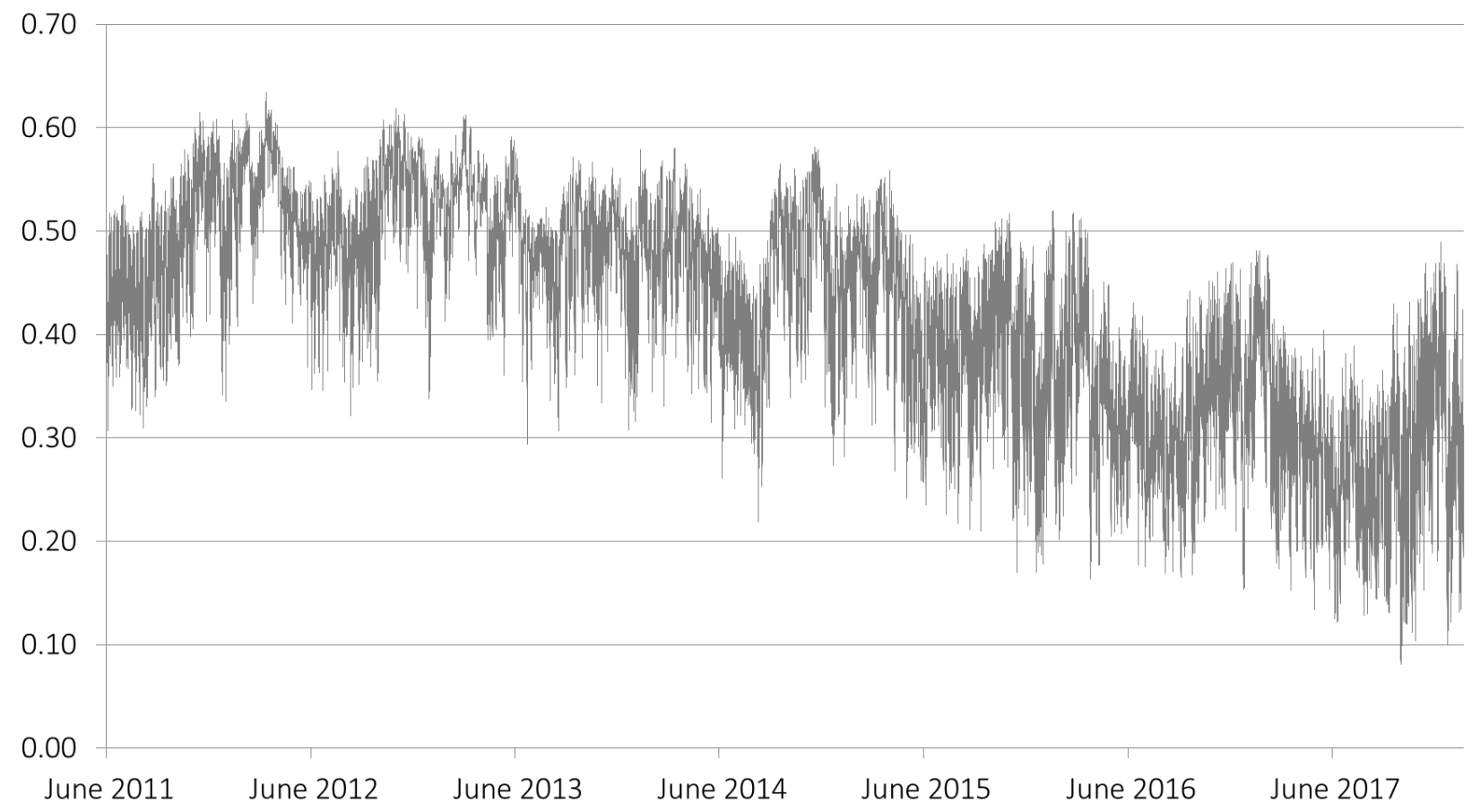

Fig.1. Mainland UK grid carbon intensity (kg/kWh)

\section{Forecast modelling}

It might be expected that carbon intensity would be related closely to the demand on the grid as the least efficient generators should be last to be dispatched. Fig. 2 shows how in one week carbon intensity is associated with demand, but that peak carbon intensity does not necessarily coincide with peak demand, so even if demand were perfectly predictable this would be inadequate for predicting carbon intensity peaks. The Pearson correlation coefficient between demand and carbon intensity for the whole data set is only 0.66, and thus carbon intensity warrants its own independent modelling. 


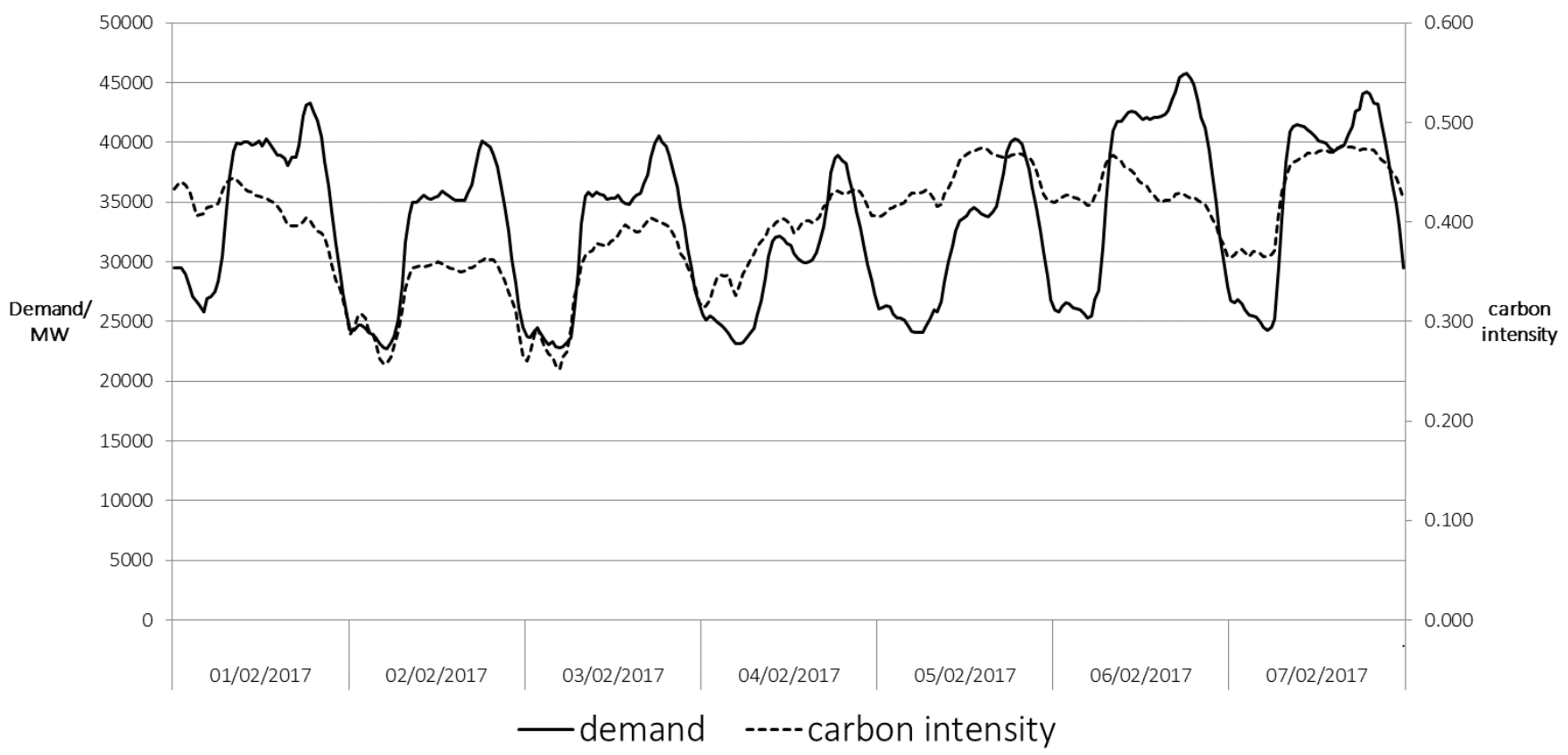

Fig. 2. Demand and carbon intensity for one week in February 2017

Initial inspection of the data indicates diurnal and weekly seasonality. A Fourier analysis of the dataset, comprising 2097 days or almost 300 weeks of data is shown in Fig. 3. Peak values occur at 2097 and 300 cycles per data-set length, i.e. daily and weekly cycles respectively. Therefore models can be expected to exploit the daily and weekly periodicity for forecasting.

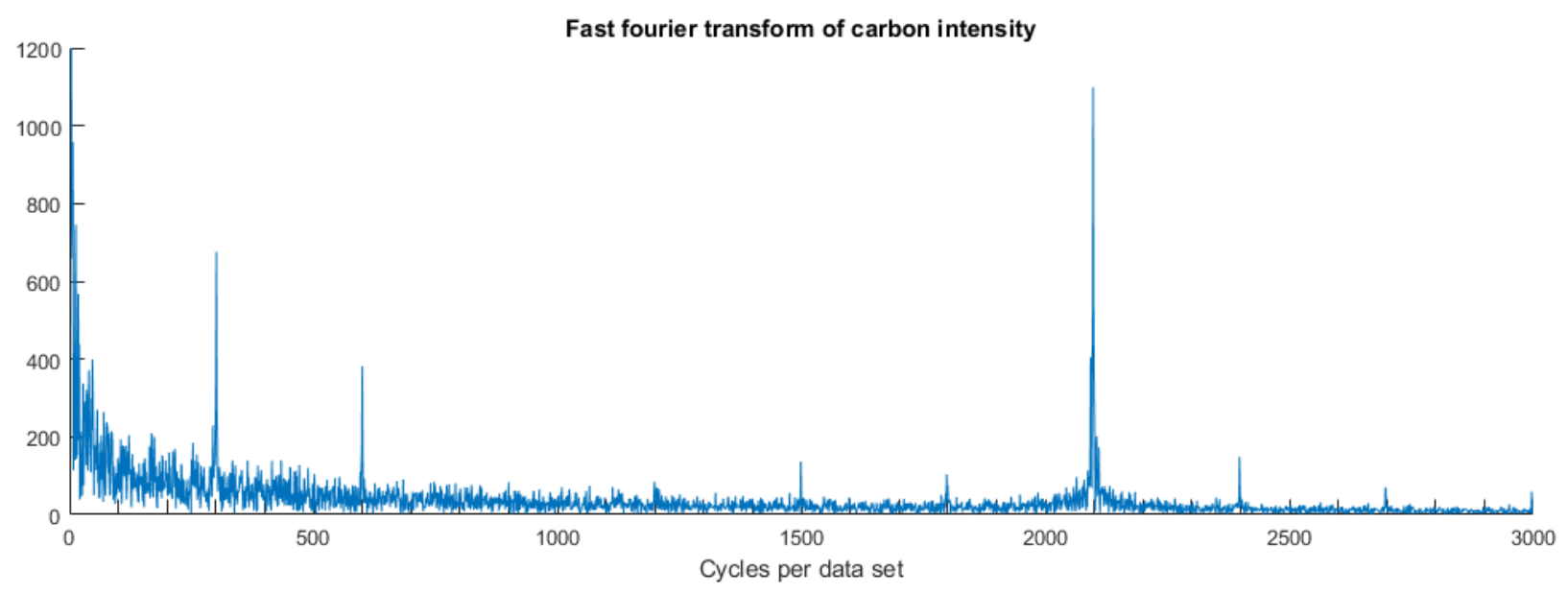

Fig. 3. Fourier transform of carbon intensity time series

In selecting the best model to fit the data, the criteria used was root mean squared error (RMSE). The intended model evolution process is to fit a linear autoregressive (AR) model to ascertain how much historical data is valuable in the predictive model. 
Then this level of detail is applied to a non-linear autoregressive model that uses a recurrent ANN. Finally, the merit of possible models is tested using the held back data, in terms of how effectively the timing of high values of carbon intensity can be forecast 24 hours ahead. Assuming this model is used to determine the timing of DR events, whether this improves carbon saving for a demand response process is evaluated.

\section{ARIMA}

Following Box et al, ${ }^{35}$ the ARIMA model takes the form shown in equation (1):

$$
\phi_{p}(B) \Phi_{P}\left(B^{\varsigma}\right)\left[\nabla^{d} \nabla_{s}^{D} y_{k}-\mu\right]=\theta_{q}(B) \Theta_{Q}\left(B^{\varsigma}\right) e_{k}
$$

where $B$ is the backshift operator and lower and uppercase functions are polynomials for the lagged and seasonally lagged time series values, respectively, and $\mu$ is the series mean. The parameters $p, d$, and $q$, and for seasonality of order $s$ the parameters $P, D$ and $Q$, determine the model structure. This is conventionally denoted $(p, d, q)(P, D, Q)_{s .}$ As can be seen from Fig. 1 the whole data series is not stationary, with a long-term downward trend illustrating the progressive grid decarbonisation. In order to fit ARIMA models, the time series should be stationary, which is conventionally achieved by differencing the data. However, in order to expose the autoregressive components that would be useful in the recurrent ANN model, a shorter data set (1 July 2016 to 30 June 2017) that is roughly stationary was used to estimate AR models. The moving average (MA) was not included in the model as the error values would not be known in time in the 48-step ahead forecasting. Therefore, in this study, parameters $d$ and D were set to zero, and polynomials $\theta$ and $\Theta$ were set to unity. The model parameters were identified using the proprietary software package SPSS v.21. The model residuals were examined for autocorrelation and the model orders, $p$ and $P$, were increased to remove any statistically significant residual autocorrelation.

\section{Diurnal seasonal model}

Initially, the autocorrelation (ACF) and partial autocorrelation functions (PACF) were examined, and the PACF showed large values for one and two lags and then weaker correlation around 48 lags. Thus, initially, the data were analysed with diurnal seasonal models, using two lagged values. Examination of the residual PACF showed significant correlation at additional lags, but these reduced significantly once the number of lags specified in the AR model was increased to four, including the seasonal autoregression. The model selected was then $(4,0,0)(4,0,0)_{48}$ with RMSE $=0.005$. For this model, the autoregressive coefficients $\left(\varphi_{\mathrm{n}}\right)$, the diurnal seasonal coefficients $\left(\Phi_{\mathrm{n}}\right)$ and the mean $(\mu)$ are shown in Table 2. This model was then used with the held back data to attempt forecasting 48 -steps. That is to say, the first to fourth lagged values were those previously predicted, but for the seasonal lagged inputs, being at least 24 hours old, actual values were used. Then forecast data 48 steps ahead is given by:

$$
\begin{aligned}
\hat{y}_{k}-\mu= & \left(\phi_{1} B+\phi_{2} B^{2}+\phi_{3} B^{3}+\phi_{4} B^{4}\right)\left(\hat{y}_{k}-\mu\right) \\
& +\left(1-\phi_{1} B-\phi_{2} B^{2}-\phi_{3} B^{3}-\phi_{4} B^{4}\right)\left(\Phi_{1} B^{48}+\Phi_{2} B^{96}+\Phi_{3} B^{144}+\Phi_{4} B^{192}\right)\left(y_{k}-\mu\right)
\end{aligned}
$$

This resulted in RMSE $=0.058$. An example of the fit for a 10-day sequence is shown in Fig. 4. 


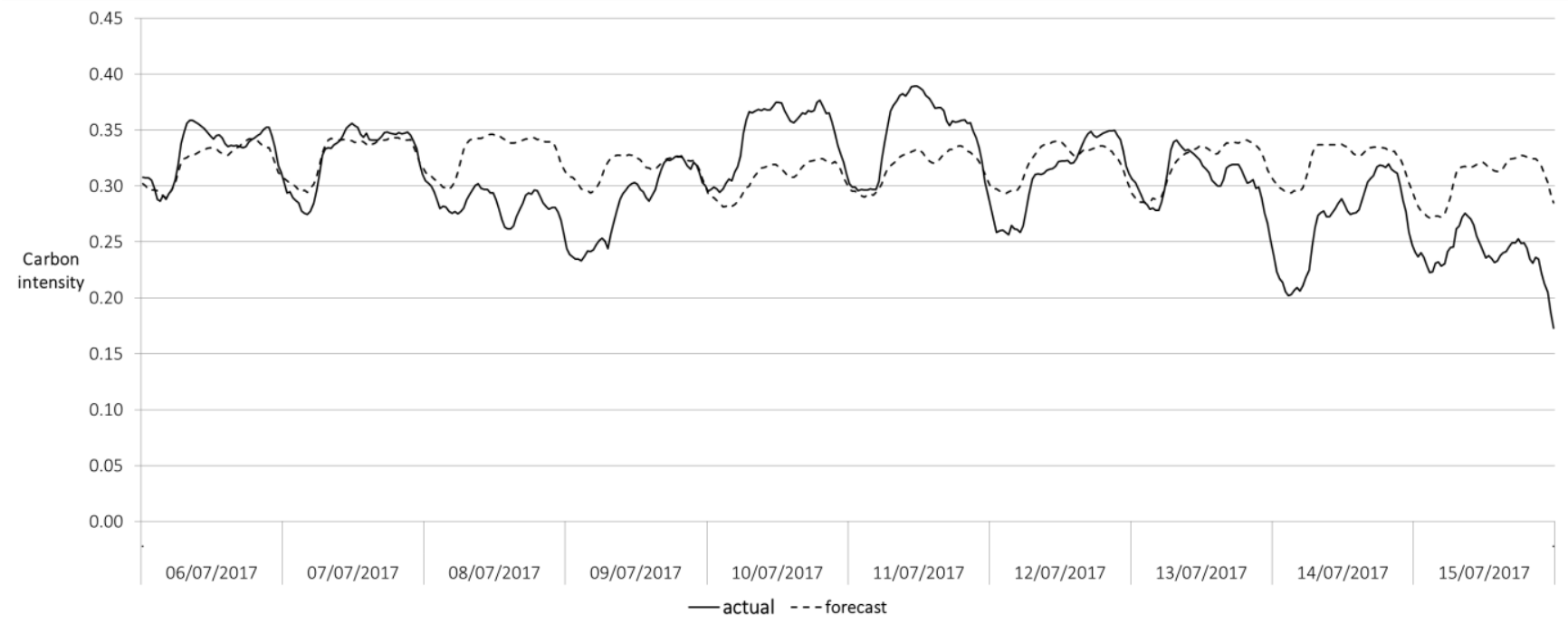

Fig. 4. Example plot for model $(4,0,0)(4,0,0)_{48}$

Weekly seasonal model

As there was evidence from the Fourier plot and the PACF of weekly seasonality, and demand patterns change at weekends, alternative AR models were tried using weekly seasonality, again using the one year's data set. The PACF showed significant correlation for up to two lags and weaker correlation at a week's lag, i.e. a lag of 336. Thus, models with two lags and varying degrees of seasonal lag were investigated and of these the model having the best fit was $(2,0,0)(5,0,0)_{336}$ with RMSE $=0.005$. So, despite the weaker partial autocorrelation for weekly then diurnal lags, the weekly seasonal model fits as well as the diurnal. The coefficients are shown in Table 2. Again this model was used for forecasting the held back data, with RMSE $=0.066$. The sequence was shorter as there was a need for a larger number of initial values to begin the forecast, and an example of the fit is shown in Fig. 5. 


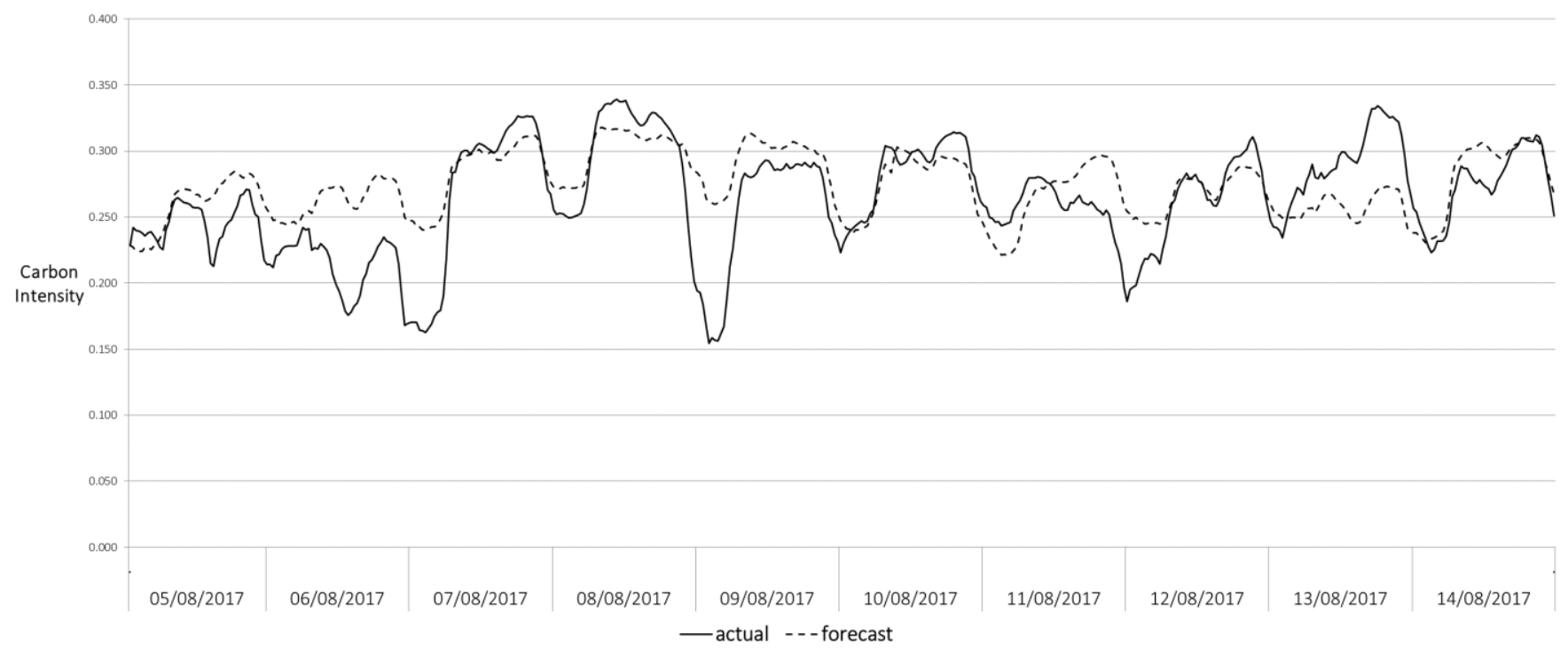

Fig. 5. Example plot for model $(2,0,0)(5,0,0)_{336}$

\begin{tabular}{|llll|}
\hline Model $(4,0,0)(4,0,0) 48$ & Model $(2,0,0)(5,0,0) 336$ \\
\hline$\mu$ & 0.319 & $\mu$ & 0.311 \\
\hline$\varphi_{1}$ & 1.529 & $\varphi_{1}$ & 1.485 \\
\hline$\varphi_{2}$ & -0.571 & $\varphi_{2}$ & -0.493 \\
\hline$\varphi_{3}$ & 0.082 & $\Phi_{1}$ & 0.233 \\
\hline$\varphi_{4}$ & -0.050 & $\Phi_{2}$ & 0.156 \\
\hline$\Phi_{1}$ & 0.266 & $\Phi_{3}$ & 0.142 \\
\hline$\Phi_{2}$ & 0.153 & $\Phi_{4}$ & 0.111 \\
\hline$\Phi_{3}$ & 0.131 & $\Phi_{5}$ & \\
\hline$\Phi_{4}$ & 0.136 & & \\
\hline Note: all significant $p<0.0005$ & & \\
\hline
\end{tabular}

Table 2. Autoregressive model coefficients 


\section{Non-linear ANN model}

The ARIMA modelling results suggest that ANN model might need to consider day-old data and week-old data up to 5 lags. Therefore these were included in the recurrent ANN model specification. The ANN model used was a three-layered network with sigmoid activation function $g$ in the hidden layer, and a linear transfer function in the output layer, and this was implemented in Matlab v.9.1. The hidden layer had five neurons for which $v_{\text {ih }}(\mathrm{i}=0,1,2, ., \mathrm{p}, \mathrm{h}=1,2, ., 5)$ are the synaptic weights for the connections between the $p$ sized input and the hidden layer, and $w_{\mathrm{h}}(\mathrm{h}=0,1,2, ., 5)$ are the synaptic weights for the connections between the hidden and the output layer. The output of the neural network from a vector of inputs $\left(\mathrm{x}_{1}, ., \mathrm{x}_{\mathrm{p}}\right)$ is:

$o=w_{o}+\sum_{h=1}^{10} w_{h} g\left(v_{o h}+\sum_{i=1}^{P} v_{i h} x_{i}\right)$

The input vector comprised previously estimated values of output up to 5 lags and then combinations of daily and weekly lagged actual values. The models with various combinations of inputs were trained on the original data set without the held back data. The models were then tested by forecasting 48-steps ahead on the held back data. For this forecasting, the ANN was used in closed-loop form so that data values lagged less than 48 steps were used as recurrent inputs. For lags greater than 48 , actual data values were used as 'independent' inputs as actual historical values would be known to the user seeking to forecast 24 hours ahead. Candidates for use in testing on the held back data were shortlisted by RMSE. The best fit model had lags in the ranges $1-4,48-52,96-$ 100 and $336-340$, thus retaining some of the diurnal and weekly dependence seen in the AR models with an estimated RMSE $=0.056$. This appears in this incidence to fit marginally better than the best AR model. An example of the fit over a ten-day period within the held back data set is shown in Fig. 6. As there is only a small difference in fit, there is no justification for favouring one modelling method. 


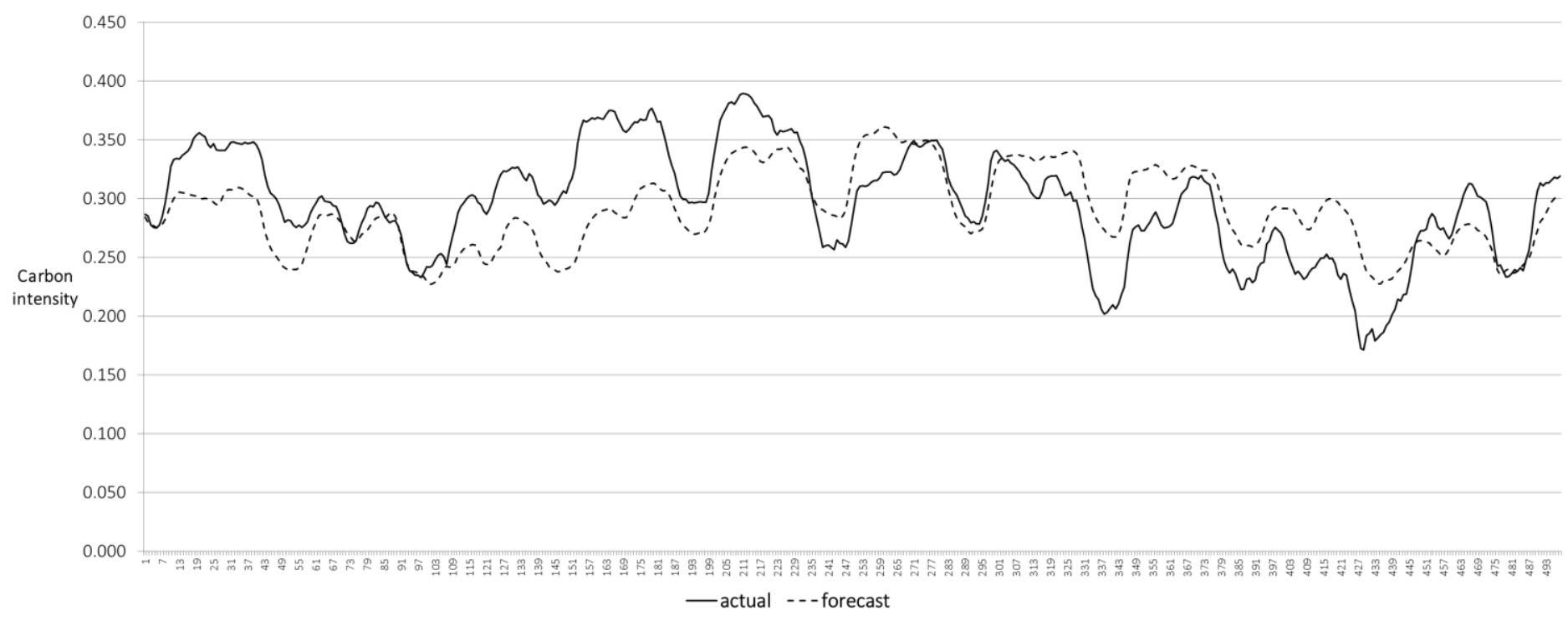

Fig. 6. Example plot using best fitting ANN model

\section{Results for carbon saving}

The models selected so far by their fit to the data were then tested for success at predicting high carbon intensity. To test the power of the different models, they were used to forecast 24 hours and thus identify the half hour in which peak carbon intensity was forecast to occur. In many cases this did not exactly coincide with the actual peak, as might be expected from the Figs. 4-6. However, a high carbon intensity half hour would still have been identified if the peak was forecast one step either side of the actual peak, as the carbon intensity does not change rapidly from one half hour to the next. As a benchmark, one might consider half-hour periods selected at random or the use of a naïve predictor that simply assumes the next 24 hours of data will be identical to the previous 24 hours. Thus a random choice would have a one in 16 , or probability of 0.06 , of coinciding with the actual peak or one step either side. The rates of success for the models are shown in Table 3, which shows no differentiation between modelling methods.

\begin{tabular}{|c|cc|}
\hline model & $\begin{array}{l}\text { rate of predicting the peak in 24 hours ahead } \\
+/ \text { - one step }\end{array}$ & RMSE \\
\hline Random choice & 0.06 & 0.060 \\
\hline Naïve predictor & 0.19 & 0.060 \\
\hline
\end{tabular}




\begin{tabular}{|llll|}
\hline AR & $(400)(400)_{48}$ & 0.25 & 0.058 \\
\hline AR & $(200)(500)_{336}$ & 0.25 & 0.066 \\
\hline ANN & $\begin{array}{l}1-4,48-52,96-100,336- \\
340\end{array}$ & 0.25 & 0.056 \\
\hline
\end{tabular}

Table 3. Rates of success in forecasting high carbon intensity

Finally, the success of these two models was assessed by calculating the carbon saving achieved by DR determined by the model's forecast of peak carbon intensity. This was calculated as the average carbon intensity of the selected half hour periods over the forecasting period. This can be compared with the average carbon intensity over the same period, and the ratio expressed as an 'improvement factor'. This period was of different length for the different models because of the different quantity of initial values each model needed. The results are shown in Table 4.

\begin{tabular}{|c|c|c|c|c|}
\hline \multirow{2}{*}{\multicolumn{2}{|c|}{ Best model }} & \multicolumn{2}{|c|}{ carbon intensity $(\mathrm{kg} / \mathrm{kWh})$} & \multirow{2}{*}{$\begin{array}{l}\text { - improvemen } \\
\text { factor }\end{array}$} \\
\hline & & \multirow{2}{*}{$\begin{array}{l}\begin{array}{l}\text { period } \\
\text { average }\end{array} \\
0.298\end{array}$} & \multirow{2}{*}{$\begin{array}{l}\begin{array}{l}\text { average } \\
\text { saved }\end{array} \\
0.358\end{array}$} & \\
\hline$A R$ & $(400)(400)_{48} f i g$ & & & 1.20 \\
\hline AR & $(200)(500)_{336}$ fig 5 & 0.303 & 0.349 & 1.15 \\
\hline ANN & $\begin{array}{l}1-4,48-52,96-100,336- \\
340\end{array}$ & 0.296 & 0.320 & 1.08 \\
\hline
\end{tabular}

Table 4. Carbon saving results for selected models

\section{Conclusion}

The use of demand response is conventionally motivated by pricing signals whereby the cost benefits of improved operation of the electricity grid are shared with consumers. In this paper it has been shown that DR activity may be undertaken to reduce carbon emissions directly. This use of DR may not provide the immediate commercial reward but could be attractive to consumers valuing short-term impact on carbon emissions. 
Many buildings use HVAC plant, which present useful loads for use in DR. It is to be expected that switching plant off needs to be planned for and, therefore, advanced warning of high carbon intensity would be useful. It has been shown that grid carbon intensity exhibits a periodicity, and this can be exploited to develop forecast models. The variation in carbon intensity can be forecast using linear autoregressive models or nonlinear ANN models. The models that best fit the time series are not necessarily best for selecting when high carbon intensity is expected to occur. The modelling methods are accessible to individual building operators as no exogenous data are used, but only data in the public domain. Using the linear diurnally seasonal AR model to determine the timing of DR can achieve an improvement in carbon emissions reduction of $20 \%$. In systems where the HVAC system load is displaced in time, the net reduction would also depend on the carbon intensity at the new operating time. This is a tool for short-term gain: increased use of DR and grid decarbonisation in the future should eventually change the pattern of daily fluctuation and reduce the incidence of high carbon intensity.

\section{Acknowledgments}

Thanks are due to initial work undertaken by Selvaganapathy Swaminathan and Paul Kamara while students at London South Bank University. This research received no specific grant from any funding agency in the public, commercial, or not-for-profit sectors.

\section{References}

1. C2ES. Outcomes of the UN Climate Change Conference in Paris. Arlington: Center for Climate and Energy Solutions, 2015.

2. DECC. Memorandum to the Energy and Climate Change Committee: Post-legislative Scrutiny of the Climate Change Act 2008 by the Secretary of State for the Department of Energy and Climate. London: H.M.S.O, 2013.

3. Terry N. Energy and carbon emissions: the way we live today. UIT, 2011.

4. Bettle R, Pout $\mathrm{CH}$ and Hitchin ER. Interactions between electricity-saving measures and carbon emissions from power generation in England and Wales. Energy Policy 2006; 34: 3434-3446. http://dx.doi.org/10.1016/j.enpol.2005.07.014

5. Hitchin R. A framework for building-related carbon coefficients and primary energy factors for networked electricity supplies. Building Serv. Eng. Res. Technol. 2017; http://dx.doi.org/10.1177/0143624417748507

6. Evans B and Sidat $\mathrm{S}$. The use of temporal factors for improved $\mathrm{CO}_{2}$ emissions accounting in buildings. Building Serv. Eng. Res. Technol. 2018; 39: 196-210 http://dx.doi.org/10.1177/0143624417753297 
7. Harmsen $\mathrm{R}$ and Graus W. How much $\mathrm{CO}_{2}$ emissions do we reduce by saving electricity? A focus on methods. Energy Policy 2013; 60: 803-812.

http://dx.doi.org/10.1016/j.enpol.2013.05.059

8. Hawkes AD. Long-run marginal $\mathrm{CO}_{2}$ emissions factors in national electricity systems. Applied Energy 2014; 125: 197-205. http://dx.doi.org/10.1016/j.apenergy.2014.03.060

9. Graff Zivin JS, Kotchen MJ and Mansur ET. Spatial and temporal heterogeneity of marginal emissions: Implications for electric cars and other electricity-shifting policies.

Journal of Economic Behavior \& Organization 2014; 107: 248-268.

http://dx.doi.org/10.1016/j.jebo.2014.03.010

10. Voorspools KR and D'haeseleer WD. An evaluation method for calculating the emission responsibility of specific electric applications. Energy Policy 2000; 28: 967980.

11. McCarthy R and Yang C. Determining marginal electricity for near-term plug-in and fuel cell vehicle demands in California: Impacts on vehicle greenhouse gas emissions. Journal of Power Sources 2010; 195: 2099-2109. http://dx.doi.org/10.1016/j.jpowsour.2009.10.024

12. Hawkes AD. Long-run marginal $\mathrm{CO}_{2}$ emissions factors in national electricity systems. Applied Energy 2014; 125: 197-205.

http://dx.doi.org/10.1016/j.apenergy.2014.03.060

13. Finenko A and Cheah L. Temporal $\mathrm{CO}_{2}$ emissions associated with electricity generation: Case study of Singapore. Energy Policy 2016; 93: 70-79.

http://dx.doi.org/10.1016/j.enpol.2016.02.039

14. Hawkes AD. Estimating marginal $\mathrm{CO}_{2}$ emissions rates for national electricity systems. Energy Policy 2010; 38: 5977-5987.

http://dx.doi.org/10.1016/j.enpol.2010.05.053

15. UKPN. Demand side response, http://innovation.ukpowernetworks.co.uk/innovation/en/research-area/demand-sideresponse/ (2016, accessed 27 July 2016).

16. Eissa MM. Demand side management program evaluation based on industrial and commercial field data. Energy Policy 2011; 39: 5961-5969.

http://doi.org/10.1016/j.enpol.2011.06.057.

17. Ayón X, Gruber JK, Hayes BP, Usaola J and Prodanović M. An optimal day-ahead load scheduling approach based on the flexibility of aggregate demands. Applied Energy 2017; 198 1-11. http://dx.doi.org/10.1016/j.apenergy.2017.04.038.

18. Motalleb M, Thornton M, Reihani E and Ghorbani R. A nascent market for contingency reserve services using demand response. Applied Energy 2016; 179: 985995. http://dx.doi.org/10.1016/j.apenergy.2016.07.078.

19. Energy Networks Association. Smart Demand Response. http://www.energynetworks.org/assets/files/news/publications/Smart_Demand_Response _A_Discussion_Paper_July12.pdf (2012, accessed 4 May 2017). 
20. Juneja S. Demand side response. London: OFGEM, 2010. Available from: https://www.ofgem.gov.uk/ofgem-publications/57026/dsr-150710.pdf (accessed 22 July 2016).

21. Pedersen TH, Hedegaard RE and Petersen S. Space heating demand response potential of retrofitted residential apartment blocks. Energy and Buildings 2017; 141: 158-166. http://dx.doi.org/10.1016/j.enbuild.2017.02.035

22. Ecotricity. Carbon content of UK Grid. https://www.ecotricity.co.uk/our-greenenergy/energy-independence/uk-grid-live (2017 accessed 25 May 2017).

23. GridCarbon, http://www.gridcarbon.uk [accessed 25.05.17].

24. UK Department for Business. Energy \& Industrial Strategy. Greenhouse gas reporting - Conversion factors 2016 , https://www.gov.uk/government/publications/greenhouse-gas-reporting-conversionfactors-2016 (2016 accessed 25 May 2017).

25. Liu K, Subbarayan S, Shoults RR, Manry MT, Kwan C and Lewis, FI. Comparison of very short-term load forecasting techniques. IEEE Transactions on Power Systems 1996; 11: 877-882. http://doi.org/10.1109/59.496169.

26. Hong WC. Electric load forecasting by support vector model. Applied Mathematical Modelling 2009; 33: 2444-2454. http://doi.org/10.1016/j.apm.2008.07.010.

27. Almeshaiei E and Soltan H. A methodology for Electric Power Load Forecasting. Alexandria Engineering Journal 2011; 50: 137-144. http://dx.doi.org/10.1016/j.aej.2011.01.015.

28. Raza MQ and Khosravi A. A review on artificial intelligence based load demand forecasting techniques for smart grid and buildings. Renewable and Sustainable Energy Reviews 2015; 50: 1352-1372. http://doi.org/10.1016/j.rser.2015.04.065.

29. Deng J. Introduction to Grey System Theory. The Journal of Grey Systems 1989; 1: $1-24$

30. Yao AWL, Chi SC and Chen JH. An improved Grey-based approach for electricity demand forecasting. Electric Power Systems Research 2003; 67: 217-224. https://doi.org/10.1016/S0378-7796(03)00112-3

31. Wang X, Qin H, Li Y, Tan Y and Cao Y. A Medium and Long-term Carbon Emission Forecasting Method for Provincial Power Grid. 2014 International Conference on Power System Technology (POWERCON 2014) Chengdu, 20-22 Oct. 2014.

32. Hsu C-C and Chen C-Y. Applications of improved grey prediction model for power demand forecasting. Energy Conversion and Management 2003; 44: 2241-2249. http://doi.org/10.1016/s0196-8904(02)00248-0.

33. Kayacan E, Ulutas B and Kaynak O. Grey system theory-based models in time series prediction. Expert Systems with Applications 2010; 37: 1784-1789.

https://doi.org/10.1016/j.eswa.2009.07.064. 
34. Pao, $\mathrm{H}-\mathrm{T}, \mathrm{Fu}, \mathrm{H}-\mathrm{C}$ and Tseng C-L. Forecasting of $\mathrm{CO}_{2}$ emissions, energy consumption and economic growth in China using an improved grey model. Energy 2012; 40: 400-409. http://doi.org/10.1016/j.energy.2012.01.037.

35. Box GEP, Jenkins GM and Reinsel GC. Time series analysis: Forecasting and control. 4th ed. United States: John Wiley \& Sons, 2008.

36. Andalib A and Atry F. Multi-step ahead forecasts for electricity prices using NARX: A new approach, a critical analysis of one-step ahead forecasts. Energy Conversion and Management 2009; 50: 739-747 http://doi.org/10.1016/j.enconman.2008.09.040.

37. Beccali M, Cellura M, Lo Brano V and Marvuglia, A. Forecasting daily urban electric load profiles using artificial neural networks. Energy Conversion and Management 2004; 45: 2879-2900. https://doi.org/10.1016/j.enconman.2004.01.006.

38. BuHamra S, Smaoui N and Gabr M. The Box-Jenkins analysis and neural networks: prediction and time series modelling. Applied Mathematical Modelling 2003; 27: 805815. https://doi.org/10.1016/S0307-904X(03)00079-9.

39. Yalcinoz T and Eminoglu U. Short term and medium term power distribution load forecasting by neural networks. Energy Conversion and Management 2005; 46: 13951405. https://doi.org/10.1016/j.enconman.2004.07.005.

40. Kumar R, Aggarwal RK and Sharma JD. Energy analysis of a building using artificial neural network: a review. Energy and Buildings 2013; 65: 352-358. http://dx.doi.org/10.1016/j.enbuild.2013.06.007.

41. Li J, Shi J and Li J. Exploring Reduction Potential of Carbon Intensity Based on Back Propagation Neural Network and Scenario Analysis: A Case of Beijing, China, Energies 2016; 9: 615. http://doi.org/10.3390/en9080615.

42. Short M, Crosbie T, Dawood M and Dawood N. Load forecasting and dispatch optimisation for decentralised co-generation plant with dual energy storage. Applied Energy 2017; 186: 304-320. http://dx.doi.org/10.1016/j.apenergy.2016.04.052.

43. Gridwatch. GB National Grid Status, http://www.gridwatch.templar.co.uk/ (2018 accessed 22 January 2018).

44. Gridwatch. UK Electricity National Grid $\mathrm{CO}_{2}$ Output per Production Type, http://gridwatch.co.uk/co2-emissions (2017 accessed 25 May 2017). 